\title{
Minireview
}

\section{Light-induced behavioral responses ('phototaxis') in prokaryotes}

\author{
Judith P. Armitage ${ }^{1, *} \&$ Klaas J. Hellingwerf ${ }^{2}$ \\ ${ }^{1}$ Department of Biochemistry, Microbiology Unit, University of Oxford, Oxford OX1 3QU, UK, ${ }^{2}$ Swammerdam \\ Institute for Life Sciences, Laboratory for Microbiology, Science Faculty, University of Amsterdam, Nieuwe Achter- \\ gracht 166, 1018 WV Amsterdam, The Netherlands; *Author for correspondence (e-mail: armitage@bioch.ox. \\ ac.uk; fax: +44-1865-275297)
}

Received 26 June 2002; accepted in revised form 16 October 2002

Key words: J. Armitage, bacteriophytochrome, C.E. Bauer, R. Clayton, chemotaxis, cyanobacteria, C. Ehrenberg, T. Engelmann, H. Gest, A. Grossman, K. Hellingwerf, photoactive yellow protein, photoreceptor, phototaxis, purple bacteria, sensory rhodopsin, J. Spudich

\begin{abstract}
Light-induced sensory responses are among the oldest scientific observations on bacterial behavior. Various types of response have been characterized physiologically in detail. However, the molecular basis of this type of response is only slowly emerging. In many of these systems photosynthetic pigments absorb the light. This then generates a signal via electron transport, feeding into a canonical chemotaxis signal transduction pathway. Nevertheless, several examples have been identified in which dedicated photoreceptor proteins do play a role. The intrinsic complexity of some of these signal transduction systems is overwhelming, in part because of the significant apparent redundancy. The genomics information that is now available for several model organisms (in particular Rhodobacter sphaeroides and Synechocystis sp. PCC6803) facilitates obtaining an increasingly detailed view of the molecular basis of the partial reactions that jointly form the basis of this type of elementary behavioral response.
\end{abstract}

\section{Introduction}

Initial development of the field

It was over a century after the very early descriptions by van Leeuwenhoek before bacteria were again studied and these were usually photosynthetic species, probably because they are often large and easily isolated from rivers and ponds. The first recorded observation of flagella in vivo was by a German traveler and observer, Christian Ehrenberg, most certainly of the purple sulfur bacterium Chromatium. He described 'wave-shaped' organelles that he thought were probably required for motility (Ehrenberg 1883). Thomas W. Engelmann isolated bacteria from the river Rhine outside his laboratory window and identified both aerotactic and chemotactic behavior, documenting the changes in swimming behavior of cells near oxygen and near carbonic acid. He suggested that bacteria could sense the difference between oxygen and carbon dioxide; something that he thought indicated the 'unity of organic nature.' In 1881 he showed that 'bacterium photometricum,' probably again a species of Chromatium, could accumulate in a light spot created on a darkened, sealed slide under certain conditions (Ehrenberg 1883; Engelmann 1883). He measured cells swimming at $20-40 \mu \mathrm{m} \mathrm{s}^{-1}$, rotating around their long axis at about $3-6$ revolutions $\mathrm{s}^{-1}$ primarily in one direction but occasionally briefly reversing. When he shone a spectrum of intense light from a gas flame through a layer of Chromatium, they accumulated in specific regions of the spectrum. A major accumulation was outside the visible region, at 850 $\mathrm{nm}$, with a second weaker accumulation at 810 and $570 \mathrm{~nm}$ and a third in the region $550-510 \mathrm{~nm}$. There were almost no bacteria in the blue and the visible red to orange regions. Watching the bacteria, he described how the bacteria appeared 'frightened' and reversed 
when they swam over either the light/dark boundaries of the light spot, or the yellow/red or far-red/red boundaries, but they showed no reaction when moving in the opposite direction. The accumulations matched the spectrum of the pigmented cells and he inferred from the coincidence of the accumulation pattern and absorption in the visible regions that these bacteria must also absorb in the invisible infrared region. This was arguably the first description of the absorption spectrum of bacteriochlorophyll.

Engelmann produced the first really detailed analysis of the behavior of a photosynthetic species, showing the coincidence between the absorption spectrum of the photopigments and the behavioral response, but the field more or less stood still for the next 50 years. Manten (Clayton 1977) re-examined photoresponses, but this time in a purple non-sulfur species: Rhodospirillum rubrum. By this time the basis of bacterial photosynthesis and the absorption spectrum of bacteriochlorophyll was known and he confirmed that the photosynthetic spectrum and that for the behavioral responses were coincident.

Roderick Clayton carried out the major study in the 1950s (Clayton 1953a-c, 1958). He was to go on to make great contributions to the world of photosynthesis (see Clayton 2002), but his career started with the first quantitative study of photoresponses in the phototroph, $R$. rubrum. He provided the first quantitative data for the 'step-down' response, showing that phototrophically grown $R$. rubrum reversed repeatedly when the light intensity was reduced. But the size and duration of the response depended not only on the size of the step-down, but also on the background light intensity and the carbon source. $\mathrm{He}$ also examined the interaction between chemotactic responses and photoresponses and found that the extent of their interaction and the response also depended on the carbon source, suggesting a strong link to metabolic state. As Engelmann had decided motility and responses were linked to the identified energy source of the day, starch, so Clayton decided the responses probably depended on changes in the levels of ATP.

Then again, the field remained more or less static for the next 25 years. While our knowledge of the molecular basis of chemotaxis in Escherichia coli reached the point where it is now probably the best understood behavioral system in biology, the study of not only bacterial photoresponses, but that of the behavior of photosynthetic species in general, more or less stood still. Although some descriptive work on the behavior of certain species in response to light was published in this period, very few genetic or quantitative studies were included. Norbert Pfennig produced some excellent video film of photoresponses in $C$. vinosum (Pfennig 1968). He confirmed the observations of Engelmann that the cells reversed direction, by reversing the direction of flagella rotation, when moving from a light to a dark environment, but showed no response when moving from a dark to light, again suggesting that the response is to a step-down in light intensity. However, there was little more quantitative investigation into the behavior of phototrophs, except for cyanobacteria, until the late 1980s. Why was this the case, and why do we still know a great deal more about photoresponses of an archaeon, Halobacterium salinarium, than we do of the much more common groups of phototrophic bacteria? The problem, we now know, lies in the complex interconnection between photo-behavior and metabolic activity and the extremely complex sensory pathways of the phototrophic species in general. In addition, it seems that, as with aerotactic responses, different species have found different mechanisms for signaling changes in light intensity to the flagellar motor. We were also, to some extent, side tracked by the assumption in the 1980s that the behavior of phototrophs would be a simple variation on the theme of $E$. coli. When it was shown that chemotaxis in E. coli did not require transport or metabolism and was therefore not affected by growth conditions, the findings made by Clayton were to a large degree, very mistakenly, dismissed.

\section{The Escherichia coli paradigm}

E. coli has four different chemoreceptors, clustered at the cell poles. These control responses to a narrow range of metabolites. They are not involved in transport or metabolism and are constitutive (although two sugar binding proteins, the maltose- and the galactose binding protein, that interact with two of the chemoreceptors, are inducible). A change in the binding of a chemoeffector to one of the membrane spanning chemoreceptor proteins (MCPs or methyl accepting chemotaxis proteins) causes a structural change to be transmitted across the membrane and alter the interaction of a linker protein, CheW, with a histidine protein kinase (HPK), CheA. CheA is somewhat different from the majority of HPKs as it controls the activity of two proteins, neither of them transcription factors. One is a small single domain response regulator, $\mathrm{CheY}$, which, when phosphorylated by CheA, 
can bind the flagellar motor and cause a change in direction of rotation and thus swimming direction. The other, CheB, is a methyl esterase that, with a methyl transferase, CheR, alters the methylation-state of the cytoplasmic domain of the chemoreceptor. CheR is consitutively active while phosphorylation of $\mathrm{CheB}$ by CheA, in response to a reduction in effector binding, increases its enzymatic activity 100-fold. The methylation state of the cytoplasmic domain is altered by a change in relative activity and the structure reset to a non-signaling state (Falke et al. 1997; Armitage 1999).

In addition to resetting the signaling state of the chemoreceptors, the CheY-P signal to the motor must also be terminated. Although the E. coli CheY-P has a relatively rapid dephosphorylation rate compared to transcriptional response regulators, it is not fast enough for bacterial chemotaxis, which requires resetting the path of migration every second or so. This means the CheY signal needs terminating within this time span. Dephosphorylation is accelerated by the interaction of CheY-P with a protein, CheZ. In addition, E. coli responds to sugars transported through the PEP-dependent phosphotransferase system with evidence suggesting a direct control by the pathway of CheA phosphorylation (Lengeler and Jahreis 1996; Lux et al. 1999). Less well understood are the effects of dicarboxylic acids on the chemotactic response, both in E. coli (Prasad et al. 1998) and in halobacteria (Montrone et al. 1996). This may constitute a separate link to cellular physiology, the so called 'energy taxis' (Taylor and Zhulin 1998; Taylor et al. 1999).

Escherichia coli does respond to changes in light intensity, even though it is not by any stretch of the imagination a photosynthetic species. A pulse of intense blue light results in a tumbling, or repellent, response that lasts for several seconds. If the intense illumination is maintained this is followed by the permanent loss of motility (and eventual cell death). Although the light intensities involved may not be physiological, these data show that light can alter the behavior of the flagellar motor. The signal involved in the blue light response is uncertain, but it seems possible that it is caused by photoexcitation of nonheme porphyrins. Whether this allows $E$. coli to escape intense blue light is unclear. An amplified response is seen in mutants impaired in the heme biosynthetic pathway (Yang et al. 1996); at moderate intensities of blue light this latter response even shows the phenomenon of adaptation.

\section{Molecular basis of light-induced behavioral responses in purple bacteria}

\section{Rhodobacter sphaeroides}

All the early data suggested that photoresponses were linked to photosynthesis. Mutants with full photosynthetic pigment complements, but no reaction center proteins, showed chemotaxis but not photoresponses (Armitage and Evans 1981). Inhibitors of electron transfer chain activity inhibited changes in membrane potential $(\Delta \phi)$ and photoresponses in the related $R$. rubrum (Harayama and Iino 1976; Harayama 1977). More recent experiments in which specific inhibitors were used to slow photosynthetic electron transport, while cells remained motile, showed that photosynthetic electron transfer is essential for photoresponses (Grishanin et al. 1997). Measurement of responses to changes in light intensity in cells grown under high and low light showed that the primary signal comes from a change in photosynthetic activity. The response only occurs when the reduction in light intensity results in the final intensity being below that needed to saturate photosynthetic electron transport. As had been seen by Clayton, the response depends not only on the size of the change in light intensity but also on the starting intensity, and it must, therefore, depend directly on photosynthetic activity rather than any specific receptors.

Photosynthetic electron transport shares components with both aerobic and anaerobic respiratory pathways. Early behavioral studies had shown that photosynthetic bacteria moved away from air bubbles when illuminated, suggesting some sort of integration between respiration and photosynthesis. $R$. sphaeroides shows a positive response to the anaerobic electron acceptor dimethyl sulphoxide (DMSO) but only if incubated anaerobically in the dark. The response depends on the enzyme DMSO reductase, but even when the enzyme is present and functional, if oxygen or light is present, electron transport to the DMSO reductase and thus chemotaxis to DMSO is lost (Gauden and Armitage 1995). As suggested in the very early experiments, signals from light and oxygen interact. Photosynthetic $R$. sphaeroides shows a stop response to a step-down in light intensity. However, if oxygen is present, the response is greatly reduced, suggesting that electron transfer to the terminal oxidases reduces the photosynthetic electron transfer rate and thus the tactic signal. Similarly, the addition of 
oxygen to light incubated cells will cause a brief response, suggesting that the oxygen has briefly reduced the rate of photosynthetic electron transfer and this has resulted in a response. This interaction of electron transfer chains should result in bacteria only being attracted to environments supporting the maximum rate of electron transfer, whatever that environment may be. Physiologically it could mean that bacteria would not move up a light gradient into an aerobic (and therefore toxic) environment as the oxygen would reduce the rate of electron transfer and result in a reversal or stop, and reorient the cells back to a light, but anoxic, environment (Armitage 1997).

Whether free-swimming cells would be able to move far enough given their inherent curved swimming paths and spontaneous direction changing every second, to sense a change in light intensity in open waters is unclear. Experiments examining the behavior of $R$. sphaeroides and Rhodospirillum centenum when resuspended in liquid medium suggest that while $R$. centenum can sense the direction of light in a dense colony, it is not able to do that in clear liquid media (Sackett et al. 1997). As with $R$. centenum, the gliding cyanobacterium Synechocystis strain PCC6803 can sense the direction of light (see below), suggesting that the changes in light intensity may be sensed in colonies and possibly therefore also in microbial mats.

What signal is sent from the electron transport chain to the bacterial flagellar motor? Over the years there has been speculation as to whether the signal for so-called 'energy taxis' might be a change in the $\Delta \mathrm{p}$, via a 'protometer' sensing changes in the proton motive force directly (Taylor 1983; Glagolev 1984), or a change in rate of electron transfer, i.e., a redox sensor. In $E$. coli aerotactic behavior is signaled primarily via the Aer protein, which has the C-terminal domain of a membrane-spanning chemosensory MCP (Bibikov et al. 1997, 2000; Rebbapragada et al. 1997) and an FAD-binding PAS domain at the N-terminus. This suggests a mechanism for sensing the changes in rate of respiration (i.e., rate of electron transfer). There is some evidence for additional $\Delta p$ sensing though an MCP, Tsr. The Aer receptor signals directly to the chemosensory pathway to control the flagellar motor. The chemosensory pathway of $R$. sphaeroides is also required for its photoresponses, suggesting the existence of a receptor to detect changes in (the rate of) photosynthetic electron transport. Indeed, measurement of the response kinetics to either a $200 \mathrm{~ms}$ step up or step down in light intensity, shows a classical response profile. This stimulus elicits a change in motor bias for $200 \mathrm{~ms}$, followed by a return to normal behavior, with a brief overshoot during about $2 \mathrm{~s}$ (Berry and Armitage 2000). This profile is the classical profile modeled on chemoreceptor-dependent responses by D. Bray and co-workers and points to the involvement of a receptor responding to changes in photosynthetic activity (Morton-Firth et al. 1999). Interestingly, the study also measured the bias of the $R$. sphaeroides motor and showed that unlike E. coli, which has a bias of 0.5 and therefore shows strong responses to both addition and removal of attractant, the bias in $R$. sphaeroides is 0.85 . This explains why the strongest responses are observed to the removal of attractant.

The genome of $R$. sphaeroides has been sequenced and annotation is ongoing. The sequence has revealed that there are three complete sets of chemoreceptor genes, encoding a total of 13 chemoreceptor proteins (4 of them putative cytoplasmic proteins): 4 CheWs, 4 CheAs, 7 CheYs, 3 CheRs, 2 CheBs and not a single CheZ. In addition there is a gene encoding a CheRBA fusion protein with a putative PAS domain (Figure 1). Initial studies involving in-frame gene deletions suggest that $\mathrm{Che}_{2}$ and $\mathrm{CheW}_{2}$ are essential for photoresponses (Romagnoli and Armitage 1999). We have also shown that $\mathrm{CheW}_{3}$ in the same operon is only required for chemotaxis under photoheterotrophic conditions. Interestingly, expression of the operon encoding $\mathrm{CheA}_{2}, \mathrm{CheW}_{2}$ and $\mathrm{CheW}_{3}$ is regulated by the Prr (Reg in $R$. capsulatus) system.

PrA, a response regulator whose activity is directly controlled via histidine protein kinase PrrCB, in response to electron flow through the constitutive terminal cytochrome $\mathrm{cbb}_{3}$ (for further details, see $\mathrm{Oh}$ et al. 2000; Oh and Kaplan 2001), binds to the Che Operon 2 promotor, increasing expression under aerobic conditions. PrrB is directly involved in aerotactic responses, but not phototactic responses (S. Romagnoli and J.P. Armitage, unpublished). The requirement for the chemosensory pathway and the response kinetics of the flagellar motor to a transient step down in light suggests that a sensory protein must signal from the electron transport chain to the chemosensory system. Work is continuing to identify this link.

\section{Mechanism of light sensing in Rhodospirillum centenum}

Colonies of $R$. centenum grown on an agar surface do show real movement towards and away from light 

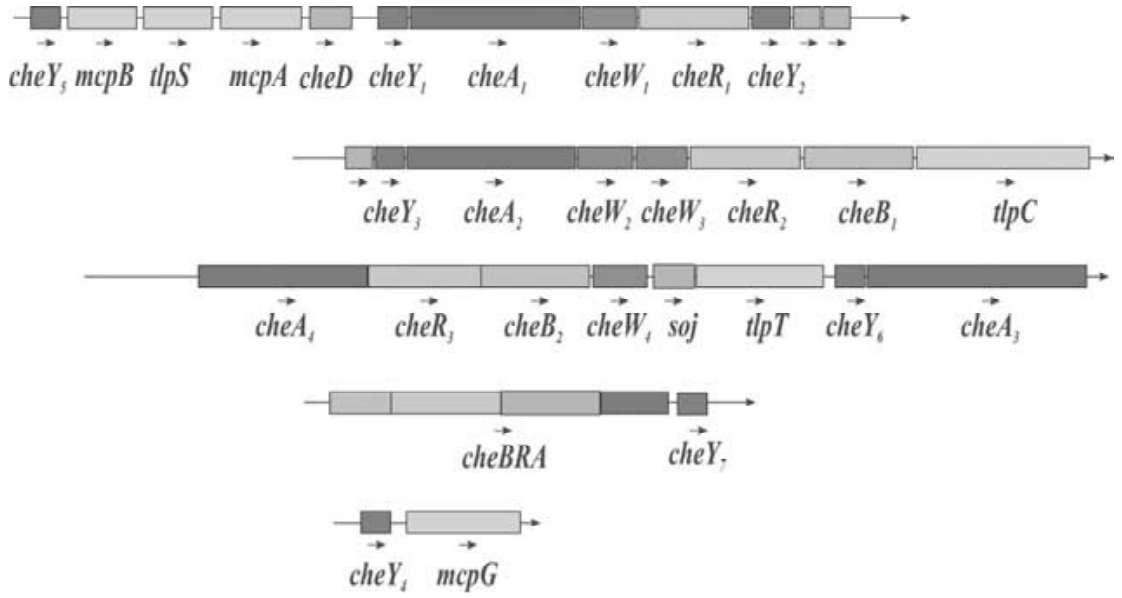

Figure 1. Chemosensory loci of Rhodobacter sphaeroides. For details of genes see text. (Tlp = transducer-like protein, receptors lacking transmembrane domains, soj $=$ homologue of partitioning protein identified in B. subtilis). Expression of the second operon is regulated by the Prr (Reg) system responsive to respiratory electron transport).
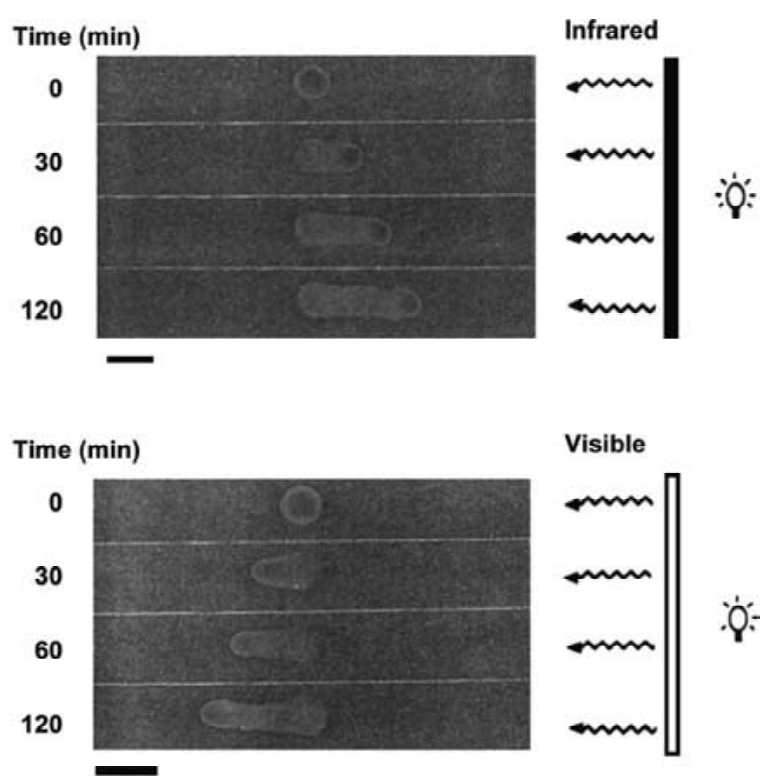

Figure 2. Photoresponses of R. centenum. Positive movement of the colony towards infra-red light, negative responses to visible light (thanks to C.E. Bauer and H. Gest, reproduced with permission). For a color version of this figure, see color section in the front of the issue.

(Ragatz et al. 1994, 1995) (Figure 2). Liquid grown $R$. centenum has a single sheathed flagellum, whose rotational direction reverses to change swimming direction. When grown on a surface, however, $R$. centenum becomes highly peritrichously flagellate, and, if the agar surface is dry enough, the colony of growing $R$. centenum will swarm slowly over the agar surface. Such colonies show both positive and negative photoresponses (called scotophobic by the authors) in a true sense, with the colony moving in a direct line towards the source of infra-red light, of wavelengths around $800-850 \mathrm{~nm}$, and away from visible light of $550-600 \mathrm{~nm}$. The observation that light wavelengths involved in positive and negative responses both include wavelengths absorbed by bacteriochlorophyll suggests that the sensory system involved must be complex, as absorption in both regions would result in increased photosynthetic activity. This colony response certainly looks like a true 'phototactic' response as described for the oriented movement of some gliding cyanobacteria. When confronted with two attractant beams of light at $90^{\circ}$ to each other the colonies moved in a direction $45^{\circ}$ between the two sources, suggesting that the directional signals were being integrated. The chemosensory pathway is essential for such photoresponses, as in $R$. sphaeroides, suggesting that the receptor signal must feed into the chemosensory pathway (Jiang and Bauer 1997; Jiang et al. 1997, 1998).

A potential photoreceptor, with strong similarity to a chemoreceptor $\mathrm{MCP}$, which probably controls the signalling to the chemosensory pathway, has been identified in $R$. centenum. This is Ptr, which is essential for both the positive (infra-red light) and negative (white light) photoresponses of the moving colonies. Ptr is structurally closely related to an MCP, and contains the highly conserved cytoplasmic signalling domain, including the conserved glutamates required for CheB/CheR dependent methylation (Jiang and Bauer 2001). Deletion of ptr results in the loss of light- 
induced methanol release associated with adaptation, as well as light dependent behaviour, but chemotaxis remains normal in such mutants. Sequence analysis identified a putative $b$-type heme-binding sequence in the periplasmic domain of Ptr. This suggests a model for photoresponses similar to that observed in the archaea, with an MCP-like protein transducing a change in light intensity and/or wavelength to the chemosensory system. In $R$. centenum, however, the light signal is converted into a change in photosynthetic electron transport, which in turn controls the redox state of the heme-binding protein and/or the putative $b$-type heme itself. The question remains, however, as to how this can produce both a negative and positive response to different wavelengths of light. Unlike the Aer protein of E. coli, Ptr has a large periplasmic domain; whether or not a second (light-sensitive) system can interact with Ptr remains to be discovered. Interestingly, analysis of the $R$. sphaeroides genome has identified two putative chemoreceptors, one membrane spanning, the other cytoplasmic, with a related motif. Experiments are under way to identify whether a similar signal relay pathway exists in this latter species.

\section{Light-induced avoidance responses in purple bacteria}

As mentioned above, in many organisms that respond to light, opposite responses are observed to intensities and/or wavelengths that are beneficial and/or detrimental to the cells, respectively. This is also true for the cyanobacteria, and even for the complex phototrophic consortia in which a central motile non-phototrophic bacterium responds to light via the surrounding phototrophic epibionts (Frostl and Overmann 2000).

Whereas in most analyses the mechanism by which purple bacteria are attracted by light is the central theme of investigations, in some organisms the avoidance response has also been characterized in detail. In $R$. centenum, this led to the discovery of a new type of MCP (i.e., Ptr, see above), involved in the lightinitiated signal transduction pathway. In Ectothiorhodospira halophila (or Halorhodospira halophila) detailed analysis of the blue-light induced avoidance response, at the single-cell level, and its adaptation to continuous illumination, has led to the identification of an entirely new class of photoreceptor proteins (Sprenger et al. 1993). The lead to this discovery was

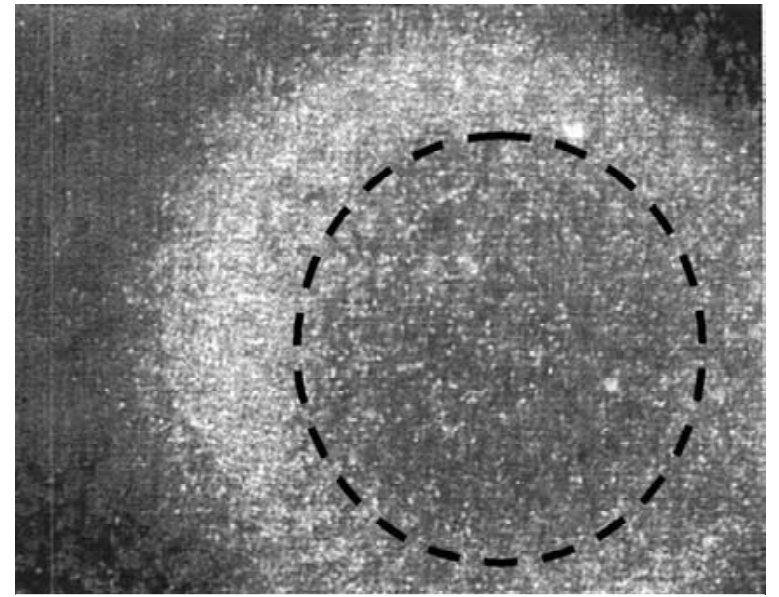

Figure 3. Accumulation pattern of cells of E. halophila in response to continuous blue light illumination. Every white spot is a single cell. The blue light was focused to cover the spot indicated by the dashed circle, prior to taking the photograph. For further details, see Sprenger et al. (1993).

the observation that - in contrast to red and infrared light, which attracts the organism - blue light acts upon it as a repellent (see, e.g., Figure 3). The underlying photoreceptor responsible for this response is the photoactive yellow protein, the primary member of the new family of the xanthopsins (Kort et al. 1996). This protein uses photochemistry based on trans to cis isomerization in its chromophore $p$-coumaric acid. This photoactive yellow protein has subsequently become a model system for photobiophysical studies (e.g., Hellingwerf et al. 2002), although the additional components that mediate signal transfer between the yellow protein and the flagella have not as yet been identified.

Various purple bacteria contain a pyp gene (encoding a photoactive yellow protein homologue) that is similar in sequence to the photoactive yellow protein from $H$. halophila. This also applies to various strains of R. sphaeroides. However, in the latter organism the photoactive yellow protein does not appear to play a role in blue-light induced avoidance responses, because knockout mutants in the pyp gene appear to retain this response (Kort et al. 2000). Rather, in this purple non-sulfur bacterium the avoidance response that is observed shows similarity to the light-induced responses in E. coli, including the moderate adaptation. 


\section{Light-induced behavioral responses in halobacteria}

The mechanisms involved in responding to light are probably the best understood in the archaeon $\mathrm{H}$. salinarium, which combines the mechanisms used for proton translocation of bacteriorhodospin (BR) with the conserved signaling domain of an MCP. H. salinarium grows in saturating salt concentrations and when the oxygen levels are low it induces BR, a retinal-based light-driven proton pump. In addition to BR $H$. salinarium has three other retinal based light absorbing pigments; halorhodopsin, HR, uses light to drive a chloride pump, but the other two are sensory rhodopsins. SR II is a constitutive retinal-containing sensory protein that absorbs blue light and undergoes a fast photocycle, generating a signal to control the flagella bundle. Increasing intensities of blue light cause the cells to reverse direction and keep the cells out of damaging blue light. Under anaerobic conditions when BR is induced, an additional sensory rhodopsin called SR I is also induced. SR I acts to produce both a positive signal in orange light and a negative repellent signal in blue light (Spudich 1985). The photopigment undergoes a fast transformation to a blue-absorbing form in orange light. If blue light is present there is a fast transformation back to the orange-absorbing form, but if blue light is not present the transformation is slow. Therefore in orange light, which is useful for BR activity, the SR I is in the blue form and sends a positive signal, but when blue light is present the orange form predominates and this sends a negative signal (for reviews see Hoff et al. 1997; Spudich 1998). Each SR protein has an accompanying sensory protein, Htr I and Htr II, respectively. These proteins turn out to have cytoplasmic domains homologous to those of the highly conserved domains of MCPs (Yao and Spudich 1992; Ferrando et al. 1993). If both of the Htrs are deleted from $H$. halobium the SR proteins are able to pump protons, just like the Schiff's base driven BR, but the presence of Htr prevents proton pumping (Bogomolni et al. 1994). Absorption of light of the appropriate wavelength leads to an electron redistribution and a configurational change in the retinal chromophore. This is transmitted to the transmembrane alpha helices of the Htr proteins, and thus to the signaling domain which controls the activity of CheA and CheY. Recent 2D crystallization studies suggest that the archaeal rhodopsins found in transport and signaling systems all use the same retinal-driven changes in protein conformation (Spudich 1998).

It was assumed for a time that this retinal based sensing system was only to be found in the extremophilic archeal species. Recently, however, a gene encoding a retinal-containing integral membrane protein was identified in a marine eubacterium (Beja et al. 2000). Further investigations (Beja et al. 2001) have suggested that this protein may be widespread amongst a diverse population of oceanic bacteria, and while a great deal more investigation is required, these data suggest that retinal based sensory systems may allow marine bacteria to maintain themselves in optimum environments in the open oceans.

\section{Light-induced behavioural responses in cyanobacteria}

\section{Phototactic diversity}

Perhaps the most complex variety of light-induced behavioral responses is seen in the cyanobacteria. This is in part based on the large variety in cellular morphology and is coupled to the various modes of cyanobacterial motility. Bacteria migrate using several different mechanisms (for a review see McBride 2001): Many species can use (a) flagella(um) to swim through aqueous media or swarm over surfaces with relatively high humidity. In addition, several species use Type IV pili to twitch or glide over a relatively dry surface, like glass, polystyrene, etc. This latter form of motility is seen e.g., in surface growing Pseudomonas aeruginosa and in 'adventurous gliding' in Myxococcus xanthus. Synechocystis PCC6803 also glides using Type IV pili. Isolation of a range of phototaxis mutants in this species identified three loci encoding chemotaxis-like proteins. Two of these were shown to be involved in photoresponses and in pili-dependent motility. One gene was identified which would encode a protein with the $\mathrm{C}$ terminus homologous to the conserved cytoplasmic signaling domain of an $\mathrm{MCP}$ and an $\mathrm{N}$ terminus with two putative phytochrome binding domains. These findings are the first to link chemotaxis-like sensory transduction in cyanobacteria with Type IV pilus-mediated phototaxis (Bhaya et al. 2001a, b). Both in the Cytophaga-Flavobacterium group and in some cyanobacteria, a second form of gliding motility is observed that appears not to make use of Type IV pili. Instead it is speculated that mobile outer membrane proteins and/or polysaccharide 
extrusion are used to propel such cells (or even entirely different mechanism as in Mycoplasma mobile). Different types of cyanobacteria make use of these different types of migration (and even additional modes like circadian reorientation with respect to the earth's surface (Ramsing et al. 2000) and flotation in the water column, but these are beyond the scope of this review).

Based on this diverse range of mechanisms of locomotion cyanobacteria carry out a wide range of behavioral responses that are elicited by changes in the illumination conditions, be it intensity, wavelength, direction and/or its degree of polarization. Many of these responses have been characterized in the 1960s and 1970 s and have been authoritatively reviewed by Häder (1987). Three different types of responses have been distinguished. The first is photokinesis, which is the light-induced alteration of the speed of migration of an organism. The second is the photophobic response, which is a reversal of movement, a temporary stop or a directional change upon a sudden change in illumination regime (e.g., the fluence rate) and the third is phototaxis, which is the directional migration with respect to the direction of light perceived. The latter type of response is observed in organisms like Phormidium autumnale (Nultsch 1961). Although it is likely that in many of these light-induced behavioral responses in cyanobacteria the photosynthetic antenna and reaction center pigments play an important role, the molecular mechanisms underlying these photoperception and signal transduction processes have remained enigmatic. To a large part this may have been due to the redundancy in the responses to illumination conditions that cyanobacteria can make use of (Choi et al. 1999; Kondou et al. 2001).

\section{Involvement of bacteriophytochromes}

An exception to this general lack of insight into underlying molecular mechanisms is now emerging. This development started with the sequencing of the genome of Synechocystis sp. PCC6803. In the sequences obtained, a number of ORFs were identified that showed homology to plant-type phytochromes. Subsequent biochemical characterisation has led to the conclusion that the products of these ORFs (e.g., Cph1; see Hughes et al. 1997) are members of an entirely new sub-family of the phytochromes, the so-called bacterio-phytochromes (for a review, see Vierstra and Davis 2000). Various subtypes can be distinguished in this family and its members appear to be abundantly present in both phototrophic and chemotrophic bacteria.

Deletion of the genes encoding the three proteins from Synechocystis with the highest similarity with phytochromes (i.e., Cph1, Cph2 and PlpA) has revealed only a putative function for PlpA, a requirement of PlpA function for growth in blue light (Wilde et al. 1997). However, after genetically decreasing the redundancy in photoresponses in the organism (Choi et al. 1999), extensive genetic screens have resulted in several mutants specifically impaired in phototaxis (Yoshihara et al. 2000; Chung et al. 2001; Bhaya et al. 2001b). Characterization of some of the mutants obtained has revealed that this light-induced phototaxis response is based on twitching motility. Presumably a signal transduction cascade is involved that shows similarity with the chemotaxis cascade of $E$. coli (just like in a set of behavioral responses in the complex chemosensory system of $P$. aeruginosa controlling twitching motility (Mattick et al. 1996)). Furthermore, one of the genes involved (sll0041, pisJ or taxD1) encodes a bacteriophytochrome, which is a/the photoreceptor in this behavioral response, a conclusion that is based on its light-modulated protein histidine protein kinase activity.

\section{UV light-induced avoidance response in Bacillus subtilus}

Very recently, it was discovered (Delprato et al. 2001) that a combination of UV-A and UV-B light (ranging from 310 to $400 \mathrm{~nm}$ ) reversibly induces an initially homogeneously distributed population of $B$. subtilis cells to migrate into a specific ring-shaped pattern. This is observed at low light intensities, when harmful effects are not yet dominating. The strength of this response appears to correlate with the number of functional DNA repair enzymes in the cell (Delprato et al. 2001). The authors speculate that the response is mediated via chemotaxis towards waste products that the B. subtilis cells would excrete. It is relevant to note that this organism has a protein with sequence similarity to UV-A/blue light photoreceptors. Akbar et al. (2001) have shown that one of the upstream components in the stress-signaling pathway in B. subtilis that activates sigma factor $\mathrm{B}$, YtvA, has considerable sequence identity with the chromophore-binding domain of the phototropin photoreceptors from plants. Upon heterologous overexpression in E. coli the YtvA protein indeed turned out to carry a flavin-type chromophore. 

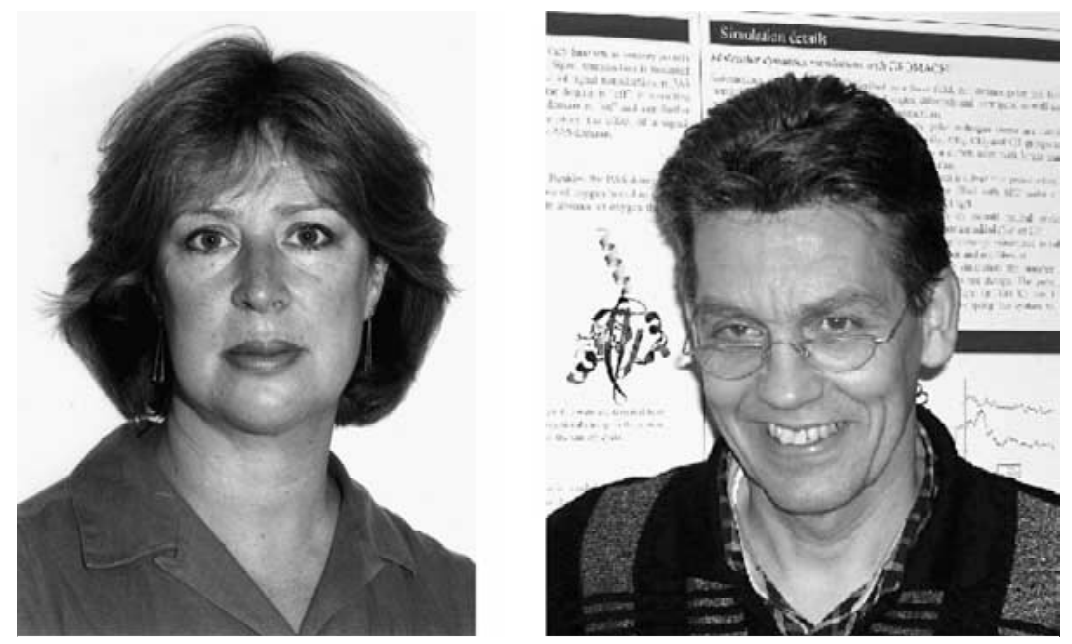

Figure 4. Judith P. Armitage (left) and Klaas J. Hellingwerf (right).

\section{The photochemical basis of photoreceptors involved in bacterial taxis}

The involvement of the photosynthesis machinery in the tactic responses in both oxyphotobacteria and anoxyphotobacteria allows for a straightforward explanation of the intimate interaction of light-induced behavioral responses and chemotactic responses (e.g., through the involvement of electron transfer and/or common redox components, see above) in many bacteria. Surprisingly, all known dedicated photoreceptors that are involved in signal generation for behavioral responses in bacteria base their photochemistry on the E/Z-isomerisation (Hellingwerf 2000) of their color-generating chromophore. This holds for the sensory rhodopsins (which now also appear to have been discovered in cyanobacteria, see for instance: http://www.grc.uri.edu/programs/2002/photos en.htm), for the bacteriophytochromes, and also for the photoactive yellow protein, a member of the Xanthopsin family.

In recent years, in eukaryotic microorganisms, plants and animals, new families of blue-light photoreceptors have been discovered in which cofactors that cannot photoisomerize, like different flavin derivatives, play a role. The best-known examples of these are the members of the cryptochrome and the phototropin family. Although scarce, a representative of these families has recently been described in the cyanobacterium Synechocystis sp. PCC6803 (Hitomi et al. 2000). As the flavin chromophore in cryptochrome can take on various redox states, this opens up the possibility that redox regulation of light wavelength sensitivity of these photoreceptor proteins may occur through coupling to the (intra) cellular redox conditions and hence to cellular physiology. Clearly, further interesting investigations lie ahead.

\section{Acknowledgments}

Research in the JPA lab is supported by the UK Biotechnology and Biological Sciences Research Council. Work in the KJH lab has been supported by the Netherlands Organization for Scientific Research (NWO). At the request of the editors, Figure 4 shows a photograph of the authors. This paper was edited by Tom Beatty.

\section{References}

Akbar S, Gaidenko TA, Kang CM, O'Reilly M, Devine KM and Price CW (2001) New family of regulators in the environmental signaling pathway which activates the general stress transcription factor sigma(B) of Bacillus subtilis. J Bacteriol 183: 1329-1338

Armitage JP (1997) Behavioural responses of bacteria to light and oxygen. Arch Microbiol 168: 249-261

Armitage JP (1999) Bacterial tactic responses. Adv Microb Physiol 41: 229-289

Armitage JP and Evans MCW (1981) The reaction centre in the phototactic and chemotactic responses of Rhodopseudomonas sphaeroides. FEMS Microbiol Lett 11: 89-92

Beja O, Aravind L, Koonin EV, Suzuki MT, Hadd A, Nguyen LP, Jovanovich SB, Gates CM, Feldman RA, Spudich JL, Spudich EN and DeLong EF (2000) Bacterial rhodopsin: evidence for a new type of phototrophy in the sea. Science 289: 1902-1906

Beja O, Spudich EN, Spudich JL, Leclerc M and DeLong EF (2001) Proteorhodopsin phototrophy in the ocean. Nature (London) 411: 786-789 
Berry RM and Armitage JP (2000) Response kinetics of tethered Rhodobacter sphaeroides to changes in light intensity. Biophys $\mathbf{J}$ 78: 1207-1215

Bhaya D, Takahashi A and Grossman AR (2001a) Light regulation of type IV pilus-dependent motility by chemosensory-like elements in Synechocystis PCC6803 Proc Natl Acad Sci USA 98: 7540-7545

Bhaya D, Takahashi A, Shahi P and Grossman AR (2001b) Novel motility mutants of Synechocystis strain PCC 6803 generated by in vitro transposon mutagenesis. J Bacteriol 183: 6140-6143

Bibikov SI, Biran R, Rudd KE and Parkinson JS (1997) A signal transducer for aerotaxis in Escherichia coli. J Bacteriol 179: 4075-4079

Bibikov SI, Barnes LA, Gitin Y and Parkinson JS (2000) Domain organization and flavin adenine dinucleotide-binding determinants in the aerotaxis signal transducer Aer of Escherichia coli. Proc Natl Acad Sci USA 97: 5830-5835

Bogomolni RA, Stoeckenius W, Szundi I, Perozo E, Olson KD and Spudich JL (1994) Removal of transducer Htr I allows electrogenic proton translocation by sensory rhodopsin I. Proc Natl Acad Sci USA 91: 10188-10192

Choi JS, Chung YH, Moon YJ, Kim C, Watanabe M, Song PS, Joe CO, Bogorad L and Park YM (1999) Photomovement of the gliding cyanobacterium Synechocystis sp. PCC6803. Photochem Photobiol 70: 95-102

Chung YH, Cho MS, Moon YJ, Choi JS, Yoo YC, Park YI, Lee KM, Kang KW and Park YM (2001) ctr1, a gene involved in a signal transduction pathway of the gliding motility in the cyanobacterium Synechocystis sp. PCC6803. FEBS Lett 492: 33-38

Clayton RK (1953a) Studies in the phototaxis of Rhodospirillum rubrum. I. Action spectrum, growth in green light and Weber-law adherence. Arch Microbiol 19: 107-124

Clayton RK (1953b) Studies in the phototaxis of Rhodospirillum rubrum.II. The relation between phototaxis and photosynthesis. Arch Microbiol 19: 125-140

Clayton RK (1953c) Studies in the phototaxis of Rhodospirillum rubrum. III. Quantitative relationship between stimulus and response Arch Microbiol 19: 141-165

Clayton RK (1958) On the interplay of environmental factors affecting taxis and mobility in Rhodospirillum rubrum. Arch Microbiol 29: 189-212

Clayton RK (1977) Light and Living Matter. Vol 2: The Biological Part, p 71. Robert E. Krieger Publishing Company, Huntington, New York

Clayton RK (2002) Research on photosynthetic reaction centers from 1932 to 1987. Photosynth Res 73: 63-71

Delprato AM, Samadani A, Kudrolli A and Tsimring LS (2001) Swarming ring patterns in bacterial colonies exposed to ultraviolet radiation. Phys Rev Lett 87: 158102-1-158102-4

Ehrenberg GS (1883) Die Infusionstrierchen als vollkommene Organismen (Ed Engelmann TW), p 15. Leipzig

Engelmann TW (1883) Bakterium photometricum. Ein Beitrag zur vergleichenden Physiologie des Licht-und Farbensinnes. Pfluegers Arch Gesamte Physiol Menschen Tiere 42: 183-186

Falke JJ, Bass RB, Butler SL, Chervitz SA and Danielson MA (1997) The two-component signaling pathway of bacterial chemotaxis: a molecular view of signal transduction by receptors, kinases, and adaptation enzymes. Annu Rev Cell Dev Biol 13: 457-512

Ferrando ME, Krah M, Marwan W and Oesterhelt D (1993) The methyl-accepting transducer protein Htr I is functionally associ- ated with the photoreceptor sensory rhodopsin I in the archaeon Halobacterium salinarium. EMBO J 12: 2999-3005

Frostl JM and Overmann J (2000) Phylogenetic affiliation of the bacteria that constitute phototrophic consortia. Arch Microbiol 174: 50-58

Gauden DE and Armitage JP (1995) Electron transport-dependent taxis in Rhodobacter sphaeroides. J Bacteriol 177: 5853-5859

Glagolev AN (1984) Bacterial $\mathrm{H}^{+}$-sensing. Trends Biochem Sci 9: $397-400$

Grishanin RN, Gauden DE and Armitage JP (1997) Photoresponses in Rhodobacter sphaeroides: role of photosynthetic electron transport. J Bacteriol 179: 24-30

Hader DP (1987) Photosensory behavior in procaryotes. Microbiol Rev 51: 1-21

Harayama S (1977) Phototaxis and membrane potential in the photosynthetic bacterium Rhodospirillum rubrum. J Bacteriol 131: 34-41

Harayama S and Iino T (1976) Phototactic responses of aerobically cultivated Rhodospirillum rubrum. J Gen Microbiol 94: 173-179

Hellingwerf KJ (2000) Key issues in the photochemistry and signalling-state formation of photosensor proteins. J Photochem Photobiol B 54: 94-102

Hellingwerf KJ, Hendriks J and Gensch T (2002) On the configurational and conformational changes in photoactive yellow protein that lead to signal generation in Ectothiorhodospira halophila. J Biol Phys 28: 395-412

Hitomi K, Okamoto K, Daiyasu H, Miyashita H, Iwai S, Toh $\mathrm{H}$, Ishiura $\mathrm{M}$ and Todo $\mathrm{T}$ (2000) Bacterial cryptochrome and photolyase: characterization of two photolyase-like genes of Synechocystis sp. PCC6803. Nucleic Acids Res 28: 2353-2362

Hoff WD, Jung KH and Spudich JL (1997) Molecular mechanism of photosignaling by archaeal sensory rhodopsins. Annu Rev Biophys Biomol Struct 26: 223-258

Hughes J, Lamparter T, Mittmann F, Hartmann E, Gartner W, Wilde $A$ and Borner TA (1997) A prokaryotic phytochrome. Nature (London) 386: 663

Jiang ZY and Bauer CE (1997) Analysis of a chemotaxis operon from Rhodospirillum centenum. J Bacteriol 179: 5712-5719

Jiang ZY and Bauer CE (2001) Component of the Rhodospirillum centenum photosensory apparatus with structural and functional similarity to methyl-accepting chemotaxis protein chemoreceptors. J Bacteriol 183: 171-177

Jiang ZY, Gest H and Bauer CE (1997) Chemosensory and photosensory perception in purple photosynthetic bacteria utilize common signal transduction components. J Bacteriol 179: 5720 5727

Jiang ZY, Rushing BG, Bai Y, Gest H and Bauer CE (1998) Isolation of Rhodospirillum centenum mutants defective in phototactic colony motility by transposon mutagenesis. J Bacteriol 180: 1248-1255

Kondou Y, Nakazawa M, Higashi S, Watanabe M and Manabe K (2001) Equal-quantum action spectra indicate fluence-rateselective action of multiple photoreceptors for photomovement of the thermophilic cyanobacterium Synechococcus elongatus. Photochem Photobiol 73: 90-95

Kort R, Crielaard W, Spudich JL and Hellingwerf KJ (2000) Colorsensitive motility and methanol release responses in Rhodobacter sphaeroides. J Bacteriol 182: 3017-3021

Kort R, Hoff WD, Van West M, Kroon AR, Hoffer SM, Vlieg KH, Crielaard W, Van Beeumen JJ and Hellingwerf KJ (1996) The xanthopsins: a new family of eubacterial blue-light photoreceptors. EMBO J 15: 3209-3218 
Lengeler JW and Jahreis K (1996) Phosphotransferase systems or PTSs as carbohydrate transport and as signal transduction systems. In: Konings WN, Kaback HR and Lolkema JS (eds) Handbook of Biological Physics, pp 573-598. Elsevier Science, Amsterdam

Lux R, Munasinghe VR, Castellano F, Lengeler JW, Corrie JE and Khan S (1999) Elucidation of a PTS-carbohydrate chemotactic signal pathway in Escherichia coli using a time-resolved behavioral assay. Mol Biol Cell 10: 1133-1146

Mattick JS, Whitchurch CB and Alm RA (1996) The molecular genetics of type-4 fimbriae in Pseudomonas aeruginosa - a review. Gene 179: 147-155

McBride MJ (2001) Bacterial gliding motility: multiple mechanisms for cell movement over surfaces. Annu Rev Microbiol 55: 49-75

Montrone M, Oesterhelt D and Marwan W (1996) Phosphorylationindependent bacterial chemoresponses correlate with changes in the cytoplasmic level of fumarate. J Bacteriol 178: 6882-6887

Morton-Firth CJ, Shimizu TS and Bray D (1999) A free-energybased stochastic simulation of the Tar receptor complex. J Mol Biol 286: 1059-1074

Nultsch W (1961) Der Einfluss des Lichtes auf die Bewegung der Cyanophyceen. 1. Phototopotaxis von Phormidium autumnale. Planta 56: 632-647

Oh JI and Kaplan S (2001) Generalized approach to the regulation and integration of gene expression. Mol Microbiol 39: 1116-1123

Oh JI, Eraso JM and Kaplan S (2000) Interacting regulatory circuits involved in orderly control of photosynthesis gene expression in Rhodobacter sphaeroides 2.4.1. J Bacteriol 182: 3081-3087

Pfennig N (1968) Chromatium akenii (Thiorhodaceae), pp 3-9. Institut für den Wissenschaftlichen Film, Göttingen, Germany

Prasad K, Caplan SR and Eisenbach M (1998) Fumarate modulates bacterial flagellar rotation by lowering the free energy difference between the clockwise and counterclockwise states of the motor. J Mol Biol 280: 821-828

Ragatz L, Jiang Z-Y, Bauer CE and Gest H (1994) Phototactic purple bacteria. Nature (London) 370: 104

Ragatz L, Jiang Z-Y, Bauer CE and Gest H (1995) Macroscopic phototactic behavior of the purple photosynthetic bacterium Rhodospirillum centenum. Arch Microbiol 163: 1-6

Ramsing NB, Ferris MJ and Ward DM (2000) Highly ordered vertical structure of Synechococcus populations within the onemillimeter-thick photic zone of a hot spring cyanobacterial mat. Appl Environ Microbiol 66: 1038-1049

Rebbapragada A, Johnson MS, Harding GP, Zuccarelli AJ, Fletcher HM, Zhulin IB and Taylor BL (1997) The Aer protein and the serine chemoreceptor Tsr independently sense intracellular energy levels and transduce oxygen, redox, and energy signals for Escherichia coli behavior. Proc Natl Acad Sci USA 94: 10541-10546
Romagnoli S and Armitage JP (1999) Role of the chemosensory pathways in transient changes in swimming speed of Rhodobacter sphaeroides induced by changes in photosynthetic electron transport. J Bacteriol 181: 34-39

Sackett MJ, Armitage JP, Sherwood EE and Pitta TP (1997) Photoresponses of the purple nonsulfur bacteria Rhodospirillum centenum and Rhodobacter sphaeroides. J Bacteriol 179: 6764-6768

Sprenger WW, Hoff WD, Armitage JP and Hellingwerf KJ (1993) The eubacterium Ectothiorhodospira halophila is negatively phototactic, with a wavelength dependence that fits the absorption spectrum of the photoactive yellow protein. J Bacteriol 175: 3096-3104

Spudich JL (1985) Bacterial Sensory Rhodopsin (SR), a Dual Attractant and Repellent Phototaxis Receptor, pp 119-127. Elsevier Science, Amsterdam

Spudich JL (1998) Variations on a molecular switch: transport and sensory signalling by archael rhodopsins. Mol Microbiol 28: 1051-1058

Taylor BL (1983) Role of proton motive force in sensory transduction in bacteria. Ann Rev Microbiol 37: 551-573

Taylor BL and Zhulin IB (1998) In search of higher energy: metabolism-dependent behaviour in bacteria. Mol Microbiol 28: 683-690

Taylor BL, Zhulin IB and Johnson MS (1999) Aerotaxis and other energy-sensing behavior in bacteria. Annu Rev Microbiol 53: 103-128

Vierstra RD and Davis SJ (2000) Bacteriophytochromes: new tools for understanding phytochrome signal transduction. Semin Cell Dev Biol 11: 511-521

Wilde A, Churin Y, Schubert H and Borner T (1997) Disruption of a Synechocystis sp. PCC6803 gene with partial similarity to phytochrome genes alters growth under changing light qualities. FEBS Lett 406: 89-92

Yang X-H, Sasarman A, Inokuchi H and Adler J (1996) Non-iron porphyrins cause tumbling to blue light by an Escherichia coli mutant defective in hemG. Proc Natl Acad Sci USA 93: 24592463

Yao VJ and Spudich JL (1992) Primary structure of an archaebacterial transducer, a methyl- accepting protein associated with sensory rhodopsin I. Proc Natl Acad Sci USA 89: 11915-11919

Yoshihara S, Suzuki F, Fujita H, Geng XX and Ikeuchi M (2000) Novel putative photoreceptor and regulatory genes required for the positive phototactic movement of the unicellular motile cyanobacterium Synechocystis sp. PCC 6803. Plant Cell Physiol 41: $1299-1304$ 